Article

\title{
Surface Characterisation of Dental Resin Composites Related to Conditioning and Finishing
}

\author{
Liliana Porojan $^{1}{ }^{\oplus}$, Roxana Diana Vasiliu ${ }^{1, *}$, Mihaela Ionela Bîrdeanu ${ }^{2}\left(\mathbb{C}\right.$ and Sorin Daniel Porojan ${ }^{3}$ \\ 1 Department of Dental Prostheses Technology (Dental Technology), Center for Advanced Technologies in \\ Dental Prosthodontics, "Victor Babeș" University of Medicine and Pharmacy, Eftimie Murgu Sq. no. 2, \\ 300041 Timisoara, Romania; sliliana@umft.ro \\ 2 National Institute for Research and Development in Electrochemistry and Condensed Matter, \\ 300569 Timisoara, Romania; mihaelabirdeanu@gmail.com \\ 3 Department of Oral Rehabilitation (Dental Technology), Center for Advanced Technologies in Dental \\ Prosthodontics, "Victor Babeș" University of Medicine and Pharmacy, Eftimie Murgu Sq. no. 2, \\ 300041 Timișoara, Romania; porojan.sorin@umft.ro \\ * Correspondence: roxana.vasiliu@umft.ro
}

Citation: Porojan, L.; Vasiliu, R.D.; Bîrdeanu, M.I.; Porojan, S.D. Surface Characterisation of Dental Resin Composites Related to Conditioning and Finishing. Polymers 2021, 13, 4236 https://doi.org/10.3390/ polym13234236

Academic Editor: Evangelia Vouvoudi

Received: 29 October 2021

Accepted: 30 November 2021

Published: 3 December 2021

Publisher's Note: MDPI stays neutral with regard to jurisdictional claims in published maps and institutional affiliations.

Copyright: (c) 2021 by the authors. Licensee MDPI, Basel, Switzerland. This article is an open access article distributed under the terms and conditions of the Creative Commons Attribution (CC BY) license (https:// creativecommons.org/licenses/by/ $4.0 /)$

\begin{abstract}
Due to the little information related to surface processing and conditioning of resin matrix ceramic materials previous glazing, the main purpose of this in vitro study was to investigate the effect of different surface treatments on the surface morphology of different resin composite materials. Five types of resin composite CAD-CAM materials: a resin composite ceramic Vita Enamic (E) and four types of nanoparticle-filled resins, like Lava Ultimate (L), Cerasmart (C), Shofu HC (S), Hyramic $(\mathrm{H})$ were taken into consideration. Specimens received the following surface treatment protocols: conventional polishing [p], polishing and glazing [pg], conditioning with CoJet [c], conditioning with CoJet and glazing [cg], sandblasting [s], sandblasting and glazing [sg], etching [e], etching and glazing [eg]. Surface roughness was analyzed for all samples and nanosurface topographic characterization was made by Atomic Force Microscopy. The highest roughness was registered for sandblasted surfaces [s], followed by tribochemical silica airborne particle abrasion [c], and etching [e]. A very strong correlated conditioning behavior of resin nanoceramic materials, like L, C and S samples was found. The microroughness decreased thus $[s]>[c]>[e]$. These are moderate correlated with $\mathrm{H}$, and are moderate negative correlated to $\mathrm{E}$, where e is more efficient. Three-dimensional images indicated visible grain boundaries after conditioning, for all materials. After polishing and glazing, surfaces became smoother. For all tested conditioning and finishing methods, surface roughness values were within clinically acceptable limits. Finishing by polishing was proved to be a good choice for all materials taken into consideration, polishing and glazing likewise, excepting Hyramic. For Enamic and Shofu HC sandblasting or tribochemical conditioning and glazing and for Hyramic polishing and glazing are not the best options, related to nanoroughness values. Referring to the nanosurface topography, for Enamic, Cerasmart and Hyramic, glazing would be the method of choice, associated with the adequate conditioning method for each material.
\end{abstract}

Keywords: atomic force microscopy (AFM); resin composite materials; surface structure

\section{Introduction}

Resin composite materials combine the properties of glass ceramics and resin composite for dental restorations [1,2]. It is well known that ceramic materials can achieve aesthetic restorations and are characterized to have high fracture resistance, but low material wear and tend to accelerate the abrasion of opposing teeth. Good processability and ease of intraoral repair are also great advantages of these materials compared to ceramics. However, polymers have poor color stability and lose surface polish after wear [1,3-8]. In this context, resin-composite materials are easy to mill, to process, to polish, have low abrasiveness, can be easy repaired, and different shade and translucency options are available [9]. As 
with all restorative nanomaterials, they are actually nanohybrids, which contain fractions of micron-sized particles. Nanoparticles along with larger particles allow a higher theoretical packing density, creating materials with fewer defects. The principal advantages of nanocomposites over other composite materials include small filler size and reduced inter-particle separation, enhanced mechanical properties, surface hardness, improved optical properties (light transmission depends on particle size), high gloss, gloss stability and excellent polishability [10-13]. Studies found a strong correlation between the surface roughness and the mechanical properties of resin composite materials [14,15]. The same study explained that high surface roughness induces pronounced grooves. Related to surface finishing, glaze firing cannot be applied because of their resin matrix components. Therefore, these materials are conventionally polished in order to obtain smooth and shiny surfaces [16], but another option is the use of a light curing glaze. Glaze materials act like sealants, to provide smoother and glossier surfaces by decreasing surface irregularities, in order to increase the wear resistance, and to improve their stain resistance [17]. Another study in literature concluded that glazing did not protect the resin composite material surfaces against surface wear [18]. Other studies in literature [19,20] focused on the various surface treatments for these materials. The findings were, that there is no universal surface treatment and further studies have to be done in this area of interest.

Roughness measurements and topography analyses are key factors in evaluating surface-oriented scientific research related to polishing and glazing. $R_{a}$ is defined by the arithmetic mean of the heights at all locations on the surface, and $R_{z}$ is defined by the mean distance between the peaks and valleys, measured in 10 points. To provide a reliable analysis of surface quality and topography, high-resolution surface roughness measurement techniques with non-contact profilometers are important. Resulted 3D surface topography maps and roughness parameters are useful for surface quality evaluations [16]. Atomic force microscopy (AFM) provides 3D topographical images of surface roughness at nanometer resolution. $S_{a}$ is defined by the arithmetic mean of the heights at all locations on the surface and $\mathrm{S}_{\mathrm{q}}$ is defined by the distance between the peaks and valleys of the sampled line, measured in the y direction [3,17-20]. Dental technicians perform glaze procedures for many types of indirect aesthetic restorations. However, together with the development of CAD/CAM processed materials, there is a trend for surface finishing by polishing. Clinicians can also polish, sandblast, condition, and glaze by themselves. However, there is a lack of studies related to the effectiveness of glaze materials, especially on current CAD/CAM restorative materials. Furthermore, CAD/CAM hybrid materials incorporate urethane dimethacrylate (UDMA) instead of bisphenol Aglycidyl methacrylate (Bis-GMA). UDMA shows higher degree of conversion and displays lower water sorption than BisGMA. Therefore, the mechanical properties of these materials are enhanced, they provided a decrease in internal defects $[13,21,22]$. Because assessment of the surface characteristics in order to obtain optimal surface treatments is essential, and due to the little information related to surface processing and conditioning of resin matrix ceramic materials previous glazing, the purpose of the study was to investigate the effect of different surface treatments on the surface morphology for reliable outcomes of finished surfaces.

The novelty of this study consists in studying five different types of dental resincomposite materials and the various conditioning and finishing methods. Some of the materials are quite new in the dental field and need more research in order to provide dental clinicians useful information. The null hypotheses were that the surface morphology of the resin-composite ceramic materials is not influenced by the conditioning method, and that there are no differences in surface characteristics between different finishing protocols.

\section{Materials and Methods}

Sample size was calculated using a software G*Power number 3.1.9.4 from University Kiel (Kiel, Germany). The effect size was chosen as 0.50 . The calculation revealed 8 samples for each group. 
Five types of resin-composite CAD-CAM Vita Enamic (VITA Zahnfabrik, Bad Säckingen, Germany) (E) and four types of nanoparticle-filled resins (Lava Ultimate, 3M ESPE, St. Paul, MN, USA) (L), (Cerasmart, GC Corporation, Tokyo, Japan) (C), (Shofu HC, Shofu, Kyoto, Japan) (S), (Hyramic Upcera, Shenyang, China) (H) were taken into consideration (Table 1). Samples were obtained by slicing the blocks in rectangular-shaped plates $(1 \mathrm{~mm}$ thick) ( $\mathrm{n}=32$ per material), resulting in 64 surfaces, polished using silicon carbide papers (600-2000 grit) and the final thickness of each specimen was checked with a digital caliper. The samples were finally manually polished using a diamond polishing paste Renfert polish all-in-one (Renfert, Hilzingen, Germany) with a low-speed handpiece. They were cleaned for 60 to $180 \mathrm{~s}$ with $98 \%$ ethylic alcohol and dried. Specimen surfaces of each material were then divided into 8 groups $(n=8)$ in terms of the applied surface treatment method: conventional polishing, polishing and glazing, conditioning with CoJet, conditioning with CoJet and glazing, sandblasting, sandblasting and glazing, etching, etching and glazing. Specimens received the following surface treatment protocols: no treatment [only polished]; $9.5 \%$ hydrofluoric acid (Yellow Porcelain Etch; Cerkamed, StalowaWola, Poland) etching for $1 \mathrm{~min}$ airborne-particle sandblasted with $50 \mu \mathrm{m}$ aluminum oxide particles (Ronvig Dental, Daugaard, Denmark) at a $10 \mathrm{~mm}$ distance for $15 \mathrm{~s}$ and tribochemical silica airborne particle abrasion with $30 \mu \mathrm{m}$ particles CoJet (3M ESPE, Seefeld, Germany) at a $10 \mathrm{~mm}$ distance for $15 \mathrm{~s}$. The sandblasting and tribochemical silica airborne particle abrasion treatments were performed by using an airborne-particle abrasion device DENTOPREP ${ }^{\text {TM }}$ Micro blaster (Ronvig Dental, Daugaard, Denmark) at 0.25 MPa. Tribochemical silica airborne particle abrasion is a chemical surface treatment. After the surface treatment protocols, the specimens were ultrasonically cleaned and degreased with $98 \%$ ethylic alcohol. Resin Glaze Primer (Shofu, Kyoto, Japan) was applied to the ceramic surfaces for $60 \mathrm{~s}$ and allowed to dry, and then two thin layers of glaze Resin Glaze Liquid (Shofu, Kyoto, Japan) were applied with a soft brush, in one direction to eliminate air bubbles and were polymerized for each $180 \mathrm{~s}$ in a light-polymerizing device SibariSr 620 (Sirio Dental, Meldola, Italy).

Table 1. Composition and manufacturer specifications of tested materials [20,21].

\begin{tabular}{ccccc}
\hline Material & Type & Monomer & Filler & Manufacturer \\
\hline Vita Enamic (E) & Resin composite & UDMA, TEGDMA & $\begin{array}{c}\text { Feldspar ceramic } \\
\text { enriched with } \\
\text { aluminum oxide 86\% }\end{array}$ & $\begin{array}{c}\text { VITA Zahnfabrik, Bad } \\
\text { Säckingen, Germany }\end{array}$ \\
\hline Lava Ultimate (L) & $\begin{array}{c}\text { CAD/CAM resin } \\
\text { composite }\end{array}$ & $\begin{array}{c}\text { Bis-GMA, UDMA, } \\
\text { Bis-EMA, TEGDMA }\end{array}$ & $\begin{array}{c}\mathrm{SiO}_{2}, \mathrm{ZrO}_{2} \text {, aggregated } \\
\mathrm{ZrO}_{2} / \mathrm{SiO}_{2} \text { cluster 80\% }\end{array}$ & $\begin{array}{c}\text { 3M ESPE, Seefeld, } \\
\text { Germany }\end{array}$ \\
\hline Cerasmart (C) & $\begin{array}{c}\text { CAD/CAM resin } \\
\text { composite }\end{array}$ & UDMA, DMA & $\begin{array}{c}\text { Silica, barium } \\
\text { glass 71\% }\end{array}$ & $\begin{array}{c}\text { GC Corporation, } \\
\text { Tokyo, Japan }\end{array}$ \\
\hline Shofu HC (S) & $\begin{array}{c}\text { CAD/CAM resin } \\
\text { composite }\end{array}$ & UDMA, TEGDMA & $\begin{array}{c}\text { Silica, silicate, } \\
\text { zirconium silicate 61\% }\end{array}$ & Shofu, Kyoto, Japan \\
\hline Hyramic (H) & CAD/CAM resin & Resin Polymers & Inorganic Filler 55-85\% & Upcera, Liaoning, China \\
\hline
\end{tabular}

\subsection{Surface Roughness Measurements}

Specimens surface roughness was analyzed in a surface profilometer Surftest SJ201 (Mitutoyo, Kawasaki, Japan). The arithmetic average roughness $\left(R_{a}\right)$ and maximum absolute vertical roughness $\left(R_{z}\right)$ [22] measurements were tested in 5 different directions and all data were registered. The mean value of the five measurements was calculated for each surface. The sampling length was $0.8 \mathrm{~mm}$, and a force of $0.7 \mathrm{mN}$ was applied.

\subsection{Nanosurface Topographic Characterization by Atomic Force Microscopy (AFM)}

Samples were examined using an atomic force microscope Nanosurf Easy Scan 2 Advanced Research (NanosurfAG, Liestal, Switzerland), in noncontact mode. Values for 
the average nanoroughness $S_{a}(n m)$ and amplitude of heights $S_{y}(n m)$ were registered. AFM investigation generated a three-dimensional image of the sample surface $(2.2 \mu \mathrm{m} \times 2.2 \mu \mathrm{m})$. AFM is a cantilever-based technique that utilizes a sharp tip to interrogate surfaces at resolutions below the optical diffraction limit. It is also a powerful tool for nano-mechanical probing and measurements.

\subsection{Statistical Analysis}

Statistical analyses were performed using the Analyse-it software (Analyse-it Software, Ltd., Leeds, UK). The unpaired $t$-test was used to evaluate the comparisons between the means. A $p$-value of under 0.05 was considered statistically significant. Spearman correlation was used to assess monotonic similar or dissimilar relationships (whether linear or not) between variables. It measures the strength of association between variables and the direction of the relationship. The significance was related to: 0.00-0.19 "very weak", 0.20-0.39 "weak", 0.40-0.59 "moderate", 0.60-0.79 "strong", 0.80-1.0 "very strong". The power of the statistical test was calculated using the software IBM SPSS (IBM, New York, NY, USA).

\section{Results}

Surface roughness values $R_{a}, R_{z}, S_{a}, S_{y}$ for conditioned surfaces are presented in Figure $1 b$.

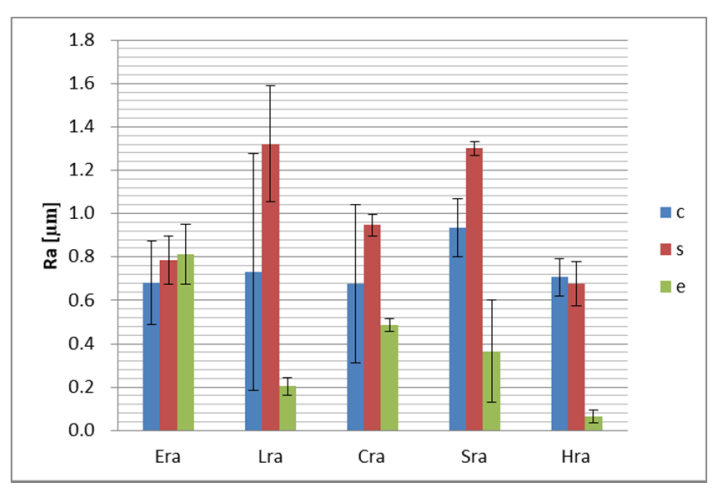

(a)

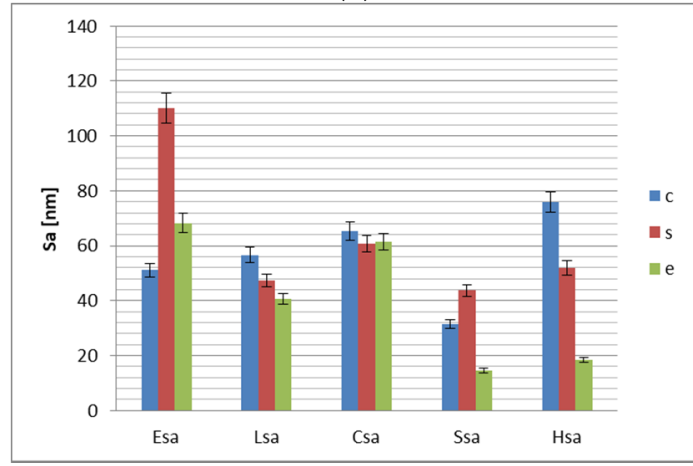

(c)

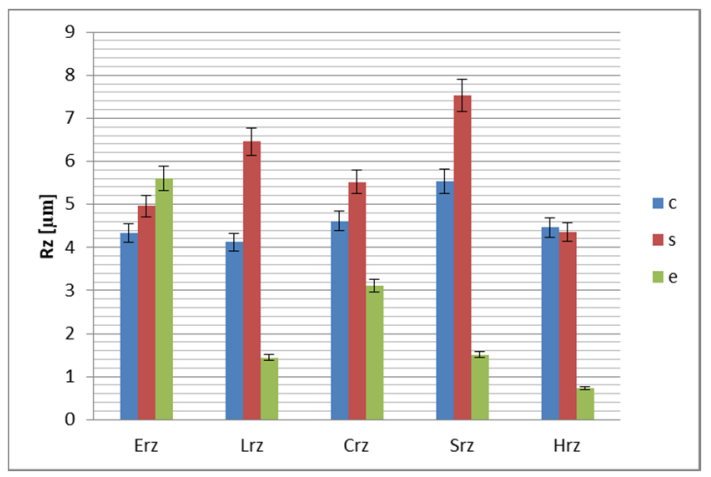

(b)

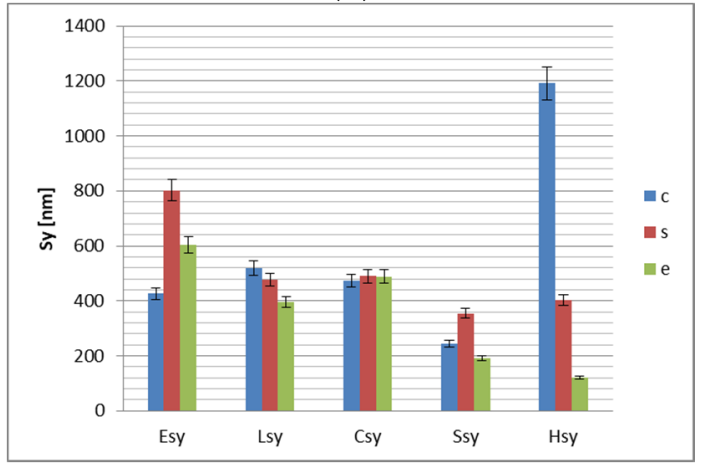

(d)

Figure 1. Surface roughness values of the samples, related to the conditioning method.(a) $R_{a}$ (average surface roughness) for the conditioned resin-composite material samples, $(b) R_{z}$ values (maximum surface roughness) for the conditioned resin-composite samples, (c) $S_{a}$ (average nanoroughness) for the conditioned resin-composite samples, (d) $S_{y}$ (amplitude of heights) for the conditioned resin-composite samples.

Relative to conditioning method, the results of the statistical analyses are presented in Tables 2-5. The highest roughness was registered for sandblasted surfaces, followed by tribochemical silica airborne particle abrasion, and etching. Significant differences 
were found between sandblasted and etched surfaces on microroughness level and for the amplitude of heights on nanolevel. On nanolevel a very strong correlation was registered between sandblasted and etched surfaces, that means that the behavior of the materials is similar to sandblasted and etched conditioning, tribochemical silica airborne particle abrasion is less efficient for $\mathrm{E}$ and more efficient for $\mathrm{H}$.

Table 2. $p$-Values related to conditioning method, after conditioning.

\begin{tabular}{ccccc}
\hline$p$-Values & $\mathbf{R}_{\mathbf{a}}$ & $\mathbf{R}_{\mathbf{z}}$ & $\mathbf{S}_{\mathbf{a}}$ & $\mathbf{S}_{\mathbf{y}}$ \\
\hline c-s & 0.071 & 0.061 & 0.664 & 0.750 \\
c-e & 0.068 & 0.089 & 0.275 & 0.391 \\
s-e & 0.035 & 0.046 & 0.053 & 0.039 \\
\hline
\end{tabular}

s (sandblasted), c (tribochemical silica airborne particle abrasion), e (etching).

Table 3. Correlation factor values related to conditioning method, after conditioning.

\begin{tabular}{ccccc}
\hline Spearman Correlation Factor & $\mathbf{R}_{\mathbf{a}}$ & $\mathbf{R}_{\mathbf{z}}$ & $\mathbf{S}_{\mathbf{a}}$ & $\mathbf{S}_{\mathbf{y}}$ \\
\hline c-s & 0.500 & 0.300 & 0.300 & 0.000 \\
c-e & 0.500 & 0.100 & 0.100 & -0.400 \\
s-e & 0.000 & 0.100 & 0.900 & 0.900 \\
\hline
\end{tabular}

s (sandblasted), c (tribochemical silica airborne particle abrasion), e (etching).

Table 4. $p$-Values related to material, after conditioning. E (Enamic), L (Lava Ultimate), H (Hyramic), $\mathrm{S}$ (Shofu), C (Cerasmart).

\begin{tabular}{ccccc}
\hline$p$-Values & $\mathbf{R}_{\mathbf{a}}$ & $\mathbf{R}_{\mathbf{z}}$ & $\mathbf{S}_{\mathbf{a}}$ & $\mathbf{S}_{\mathbf{y}}$ \\
\hline E-L & 0.984 & 0.623 & 0.287 & 0.362 \\
E-C & 0.731 & 0.627 & 0.532 & 0.351 \\
E-S & 0.746 & 0.962 & 0.079 & 0.054 \\
E-H & 0.365 & 0.371 & 0.403 & 0.932 \\
L-C & 0.818 & 0.641 & 0.055 & 0.662 \\
L-S & 0.240 & 0.169 & 0.129 & 0.044 \\
L-H & 0.292 & 0.365 & 0.970 & 0.742 \\
C-S & 0.375 & 0.719 & 0.063 & 0.041 \\
C-H & 0.289 & 0.196 & 0.471 & 0.812 \\
S-H & 0.087 & 0.156 & 0.282 & 0.438 \\
\hline
\end{tabular}

Table 5. Correlation factor values related to material, after conditioning. E (Enamic), L (Lava Ultimate), H (Hyramic), S (Shofu), C (Cerasmart).

\begin{tabular}{ccccc}
\hline Spearman Correlation Factor & $\mathbf{R}_{\mathbf{a}}$ & $\mathbf{R}_{\mathbf{z}}$ & $\mathbf{S}_{\mathbf{a}}$ & $\mathbf{S}_{\mathbf{y}}$ \\
\hline E-L & -0.500 & -0.500 & -0.500 & -0.500 \\
E-C & -0.500 & -0.500 & -1.000 & 1.000 \\
E-S & -0.500 & -0.500 & 0.500 & 0.500 \\
E-H & -1.000 & -1.000 & -0.500 & -0.500 \\
L-C & 1.000 & 1.000 & 0.500 & -0.500 \\
L-S & 1.000 & 1.000 & 0.500 & 0.500 \\
L-H & 0.500 & 0.500 & 1.000 & 1.000 \\
C-S & 1.000 & 1.000 & -0.500 & 0.500 \\
C-H & 0.500 & 0.500 & 0.500 & -0.500 \\
S-H & 0.500 & 0.500 & 0.500 & 0.500 \\
\hline
\end{tabular}

Surface roughness values $R_{a}, R_{z}, S_{a}$, $S_{y}$ for finished surfaces are presented in Figure 2. 


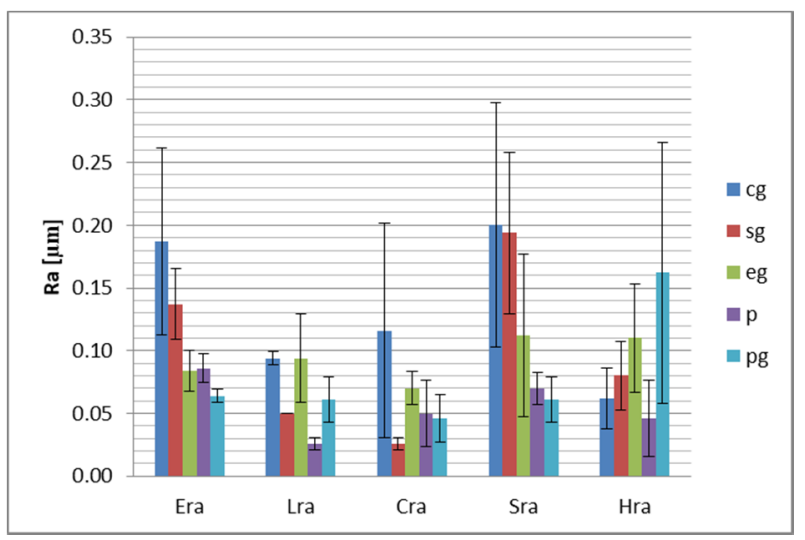

(a)

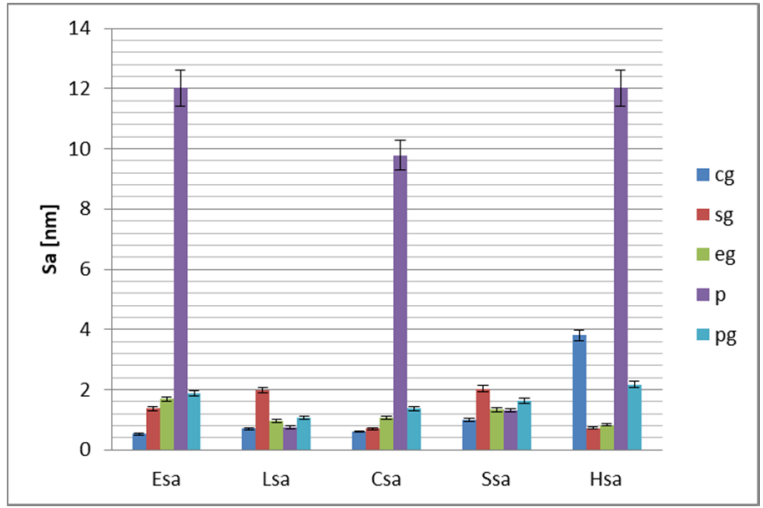

(c)

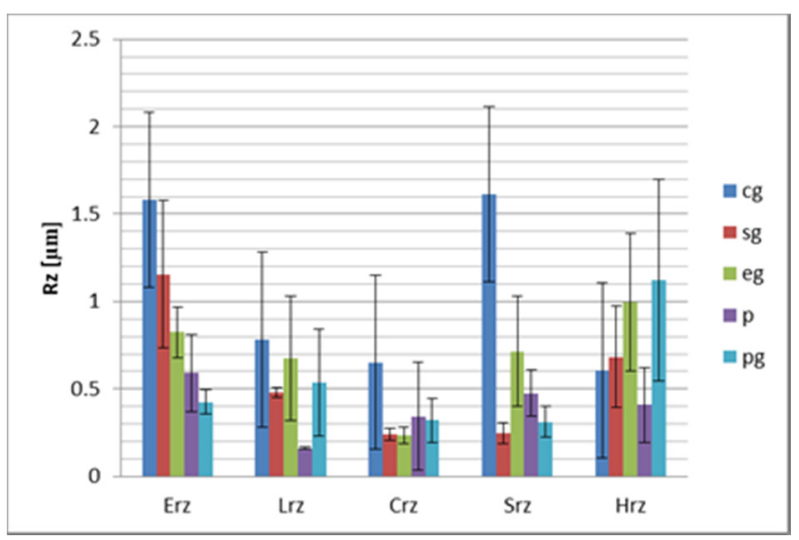

(b)

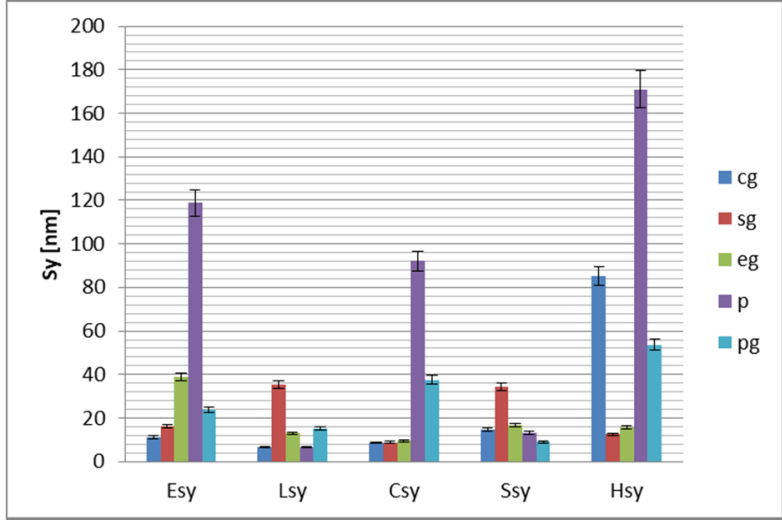

(d)

Figure 2. Surface roughness values of the samples, related to the finishing method. (a) $R_{a}$ (average surface roughness) for the finished resin-composite samples, $(\mathbf{b}) R_{\mathrm{z}}$ values (maximum surface roughness) for the finished resin-composite samples, (c) $S_{a}$ (average nanoroughness) for the finished resin-composite samples, (d) $S_{y}$ (amplitude of heights) for the finished resin-composite samples.

As a result of materials correlations records, a very strong correlation has been measured between Lava Ultimate, Cerasmart, and Shofu, relative to microroughness $\left(R_{a}\right.$ and $\mathrm{R}_{\mathrm{z}}$ ). A moderate correlation was registered between the listed materials and Hyramic, and a negative moderate correlation between these and Enamic. The correlation is very strong negative between Enamic and Hyramic. On nanolevel, the behavior is different, is strong correlated for $\mathrm{L}$ and $\mathrm{H}$. Between materials, roughness values do not vary significantly, with few exceptions, for $S_{y}$, between Lava Ultimate and Shofu, and between Cerasmart and Shofu. It can be stated that all conditioning methods are effective on all materials taken into consideration. $R_{a}$ values are $0.745 \pm 0.107 \mu \mathrm{m}$ for tribochemical silica airborne particle abrasion, $1.006 \pm 0.294 \mu \mathrm{m}$ for sandblasted surfaces and $0.383 \pm 0.287 \mu \mathrm{m}$ for etched surfaces. $S_{a}$ values are $56.052 \pm 16.664 \mathrm{~nm}$ for tribochemical silica airborne particle abrasion, $62.764 \pm 27.280 \mathrm{~nm}$ for sandblasted and $40.651 \pm 24.378$ for etched surfaces.

Related to the conditioning method, Lava Ultimate, Cerasmart and Shofu samples demonstrated a very strong correlated conditioning behavior, the miroroughness decreased thus sandblasted > tribochemical silica airborne particle abrasion > etched with a significant variation between sandblasted and etched. Previous materials are moderate correlated with Hyramic, to which in addition sandblasted and tribochemical silica airborne particle abrasion are close together and moderate negative correlated to Enamic, where etching is more efficient, all the conditioning methods are close together. That means the first null hypothesis: "the surface morphology of the resin-composite materials is not influenced by the conditioning method" was rejected. 
Relative to finishing method, the results of the statistical analyses are presented in Tables 6-9.

Table 6. $p$-Values related to conditioning method, after finishing.

\begin{tabular}{ccccc}
\hline$p$-Values & $\mathbf{R}_{\mathbf{a}}$ & $\mathbf{R}_{\mathbf{z}}$ & $\mathbf{S}_{\mathbf{a}}$ & $\mathbf{S}_{\mathbf{y}}$ \\
\hline cg-sg & 0.073 & 0.054 & 0.964 & 0.803 \\
cg-eg & 0.158 & 0.119 & 0.839 & 0.648 \\
cg-p & 0.004 & 0.007 & 0.069 & 0.033 \\
cg-pg & 0.188 & 0.123 & 0.588 & 0.764 \\
sg-eg & 0.882 & 0.309 & 0.529 & 0.702 \\
sg-p & 0.086 & 0.219 & 0.106 & 0.104 \\
sg-pg & 0.572 & 0.914 & 0.564 & 0.570 \\
eg-p & 0.016 & 0.033 & 0.076 & 0.055 \\
eg-pg & 0.339 & 0.177 & 0.113 & 0.325 \\
p-pg & 0.308 & 0.343 & 0.082 & 0.047
\end{tabular}

cg (tribochemical silica airborne particle abrasion and glazed), sg (sandblasted and glazed), pg (polished and glazed), $\mathrm{p}$ (polished).

Table 7. Correlation factor values related to conditioning method, after finishing.

\begin{tabular}{ccccc}
\hline Spearman Correlation Factor & $\mathbf{R}_{\mathbf{a}}$ & $\mathbf{R}_{\mathbf{z}}$ & $\mathbf{S}_{\mathbf{a}}$ & $\mathbf{S}_{\mathbf{y}}$ \\
\hline cg-sg & 0.771 & 0.029 & 0.200 & -0.257 \\
cg-eg & 0.087 & 0.086 & -0.700 & 0.429 \\
cg-p & 0.886 & 0.543 & -0.103 & 0.543 \\
cg-pg & -0.261 & -0.600 & 0.300 & 0.429 \\
sg-eg & 0.551 & 0.771 & 0.300 & 0.200 \\
sg-p & 0.771 & 0.543 & -0.564 & -0.829 \\
sg-pg & 0.319 & 0.600 & -0.200 & -0.829 \\
eg-p & -0.058 & 0.771 & 0.103 & 0.200 \\
eg-pg & 0.397 & 0.371 & 0.000 & -0.257 \\
p-pg & 0.029 & -0.200 & 0.872 & 0.771 \\
\hline
\end{tabular}

cg (tribochemical silica airborne particle abrasion and glazed), sg (sandblasted and glazed), pg (polished and glazed), p (polished).

Table 8. $p$-Values related to material, after finishing. E (Enamic), L (Lava Ultimate), C (Cerasmart), $\mathrm{S}$ (Shofu), H (Hyramic).

\begin{tabular}{ccccc}
\hline$p$-Values & $\mathbf{R}_{\mathbf{a}}$ & $\mathbf{R}_{\mathbf{z}}$ & $\mathbf{S}_{\mathbf{a}}$ & $\mathbf{S}_{\mathbf{y}}$ \\
\hline E-L & 0.093 & 0.081 & 0.342 & 0.307 \\
E-C & 0.052 & 0.029 & 0.110 & 0.263 \\
E-S & 0.280 & 0.223 & 0.402 & 0.322 \\
E-H & 0.632 & 0.621 & 0.606 & 0.219 \\
L-C & 0.770 & 0.174 & 0.438 & 0.447 \\
L-S & 0.083 & 0.504 & 0.017 & 0.447 \\
L-H & 0.275 & 0.126 & 0.275 & 0.186 \\
C-S & 0.080 & 0.163 & 0.530 & 0.494 \\
C-H & 0.346 & 0.081 & 0.139 & 0.100 \\
S-H & 0.454 & 0.783 & 0.322 & 0.186 \\
\hline
\end{tabular}

After surface finishing, significant differences were found for air abraised, glazed surfaces and polished surfaces and etched, glazed and polished samples on microroughness level and for air abraised, glazed surfaces and polished surfaces and polished, glazed and simply polished for the amplitude of heights, on nanolevel. Polished surfaces recorded significantly lower values compared to these. The $\mathrm{cg}$ and $\mathrm{p}$ are strong correlated for $\mathrm{R}_{\mathrm{a}}$, that means that between materials, the behavior related to these finishing methods is similar. $\mathrm{R}_{\mathrm{a}}$ values are higher for $\mathrm{cg}$ than for $p$, for all materials. Between materials, roughness values after finishing are not significant different, excepting $R_{z}$ for Enamic-Cerasmart and $S_{a}$ for Lava Ultimate-Shofu. For $R_{a}$ it is a strong correlation for Enamic-Shofu, for them the 
values are polished, glazed $<$ polished $<$ etched and glazed $<$ sandblasted and glazed. On nanolevel Enamic-Cerasmart are strong correlated.

Table 9. Correlation factor values related to material, after finishing. E (Enamic), L (Lava Ultimate), C (Cerasmart), S (Shofu), H (Hyramic).

\begin{tabular}{ccccc}
\hline Spearman Correlation Factor & $\mathbf{R}_{\mathbf{a}}$ & $\mathbf{R}_{\mathbf{z}}$ & $\mathbf{S}_{\mathbf{a}}$ & $\mathbf{S}_{\mathbf{y}}$ \\
\hline E-L & 0.051 & 0.500 & 0.100 & -0.205 \\
E-C & 0.300 & 0.200 & 1.000 & 0.900 \\
E-S & 0.900 & 0.400 & 0.100 & -0.300 \\
E-H & -0.700 & -0.400 & 0.400 & 0.300 \\
L-C & 0.667 & 0.100 & 0.100 & -0.103 \\
L-S & 0.410 & 0.700 & 1.000 & 0.359 \\
L-H & 0.410 & 0.300 & -0.800 & -0.872 \\
C-S & 0.400 & 0.400 & 0.100 & -0.600 \\
C-H & -0.300 & -0.600 & 0.400 & 0.400 \\
S-H & -0.400 & -0.300 & -0.800 & -0.700 \\
\hline
\end{tabular}

After glazing $R_{a}$ values decrease to $0.131 \pm 0.053 \mu \mathrm{m}$ for $\mathrm{cg}, 0.097 \pm 0.060 \mu \mathrm{m}$ for sandblasted and glazed and $0.094 \pm 0.015 \mu \mathrm{m}$ for etched and glazed, $0.055 \pm 0.002 \mu \mathrm{m}$ for polished, $0.078 \pm 0.004 \mu \mathrm{m}$ for polished and glazed. $S_{a}$ values are $1.327 \pm 1.394 \mathrm{~nm}$ for tribochemical silica airborne particle abrasion and glazed, $1.365 \pm 0.647 \mathrm{~nm}$ for sandblasted and glazed and $1.171 \pm 0.340$ for etched and glazed $7.178 \pm 5.689 \mathrm{~nm}$ for polished, $1.625 \pm 0.428 \mathrm{~nm}$ for polished and glazed. On nanolevel the roughness of polished surfaces is higher for Enamic, Cerasmart and Hyramic.

After finishing, all measured $R_{a}$ values are lower than $0.200 \mu \mathrm{m}$. For [polished] samples $R_{a}$ values were between 0.026 and $0.086 \mu \mathrm{m}$, for [polished and glazed] between 0.046 and $0.064 \mu \mathrm{m}$, except for Hyramic $(0.162 \mu \mathrm{m})$. For Enamic and Cerasmart glazing of polished surfaces decrease $R_{a}$ values and significant decrease $S_{a}$ values, for $S$ only $R_{a}$ values. For [etched and glazed] samples $R_{a}$ values were between 0.070 and $0.112 \mu \mathrm{m}$. Related to tribochemical silica airborne particle abrasion and glazed and sandblasted and glazed the behavior is more favorable for Lava Ultimate, Cerasmart and Hyramic.

Representative AFM images of all the materials are presented in Figures 3-7. The AFM images have a size of $2.2 \mu \mathrm{m}^{2}$. The grain boundaries were clearly visible for all materials. The grains exhibited a smooth surface with a dense network of interlocking acicular branches, where interstices are filled with a compact amorphous phase. After polishing and glazing, surfaces became smoother.

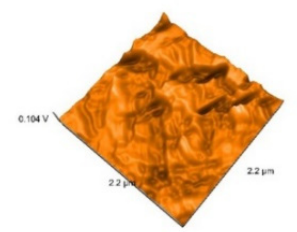

(a)

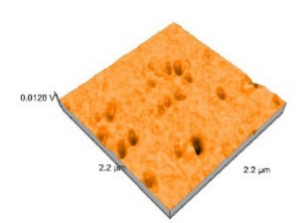

(e)

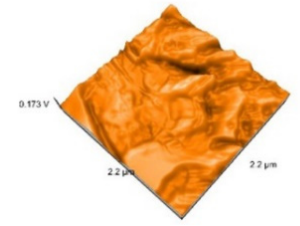

(b)

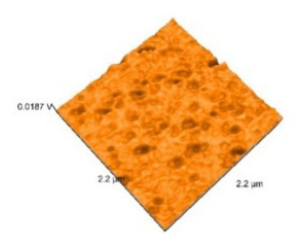

(f)

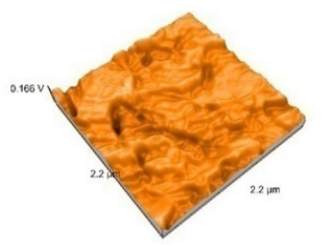

(c)

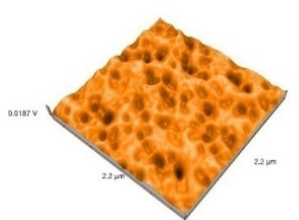

(g)

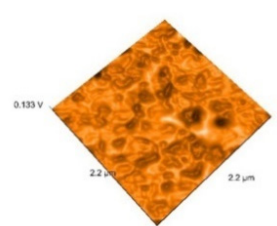

(d)

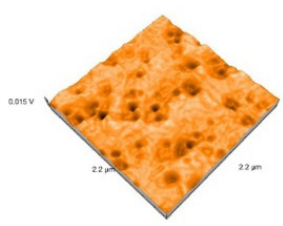

(h)

Figure 3. 3D images of Vita Enamic specimens: (a) Enamic-tribochemical silica airborne particle abrasion, (b) Enamicsandblasted, (c) Enamic-etched, (d) Enamic-polished, (e) Enamic tribochemical silica airborne particle abrasionand glazed +, (f) Enamic-sandblasted and glazed (g) Enamic etched and glazed, (h) Enamic polished and glazed. 


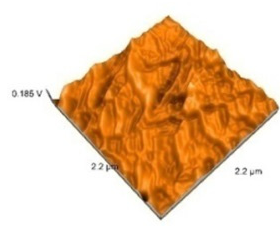

(a)

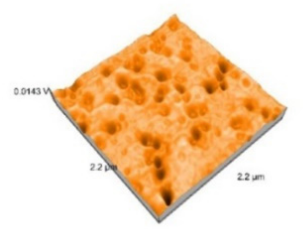

(e)

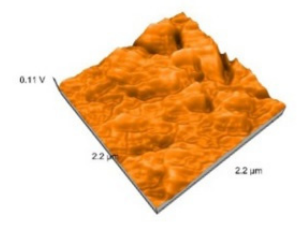

(b)

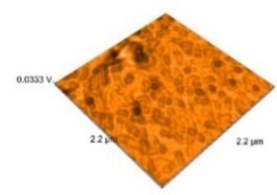

(f)

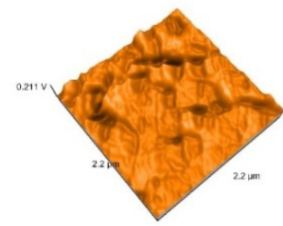

(c)

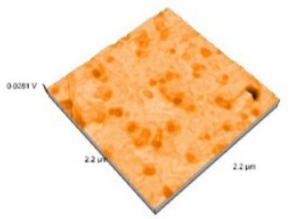

(g)

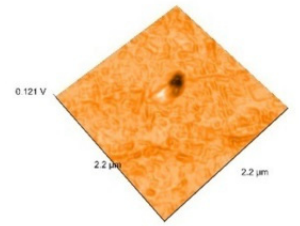

(d)

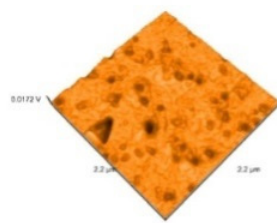

(h)

Figure 4. 3D images of Lava specimens: (a) Lava Ultimate-tribochemical silica airborne particle abrasion, (b) Lava Ultimate sandblasted, (c) Lava Ultimate etched, (d) Lava Ultimate polished, (e) Lava Ultimate airborne particle abrasion and glazed, (f) Lava Ultimate sandblasted and glazed (g) Lava Ultimate etched glazed, (h) Lava Ultimate polished glazed.

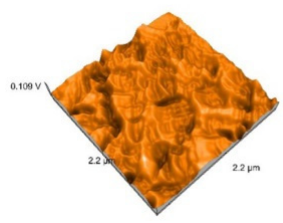

(a)

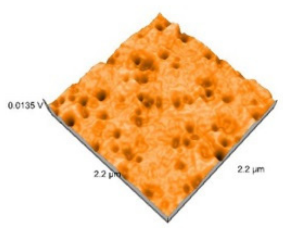

(e)

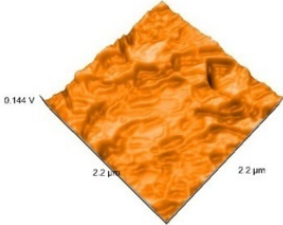

(b)

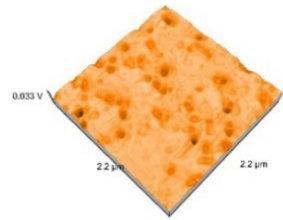

(f)

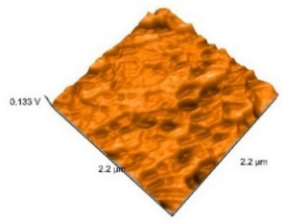

(c)

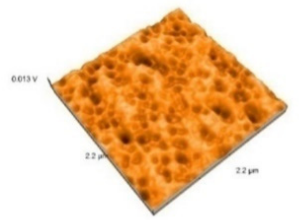

(g)

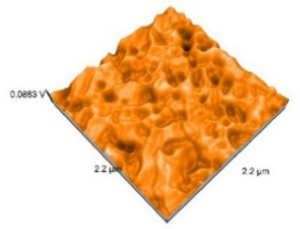

(d)

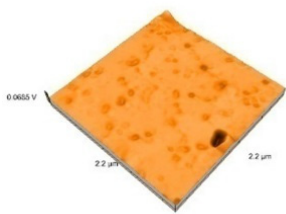

(h)

Figure 5. 3D images of Cerasmart specimens: (a) Cerasmart-tribochemical silica airborne particle abrasion (b) Cerasmart sandblasted, (c) Cerasmart etched, (d) Cerasmart polished, (e) Cerasmart-tribochemical silica airborne particle abrasion and glazed, (f) Cerasmart sandblasted glazed, (g) Cerasmart etched glazed, (h) Cerasmart polished glazed.

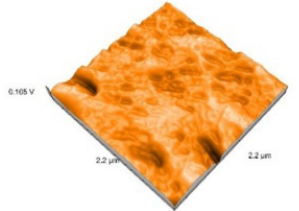

(a)

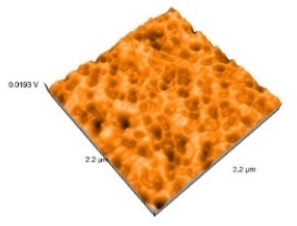

(e)

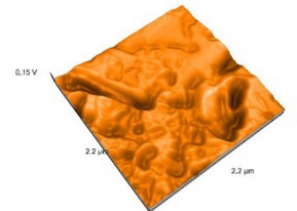

(b)

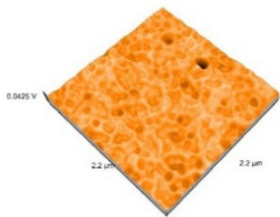

(f)

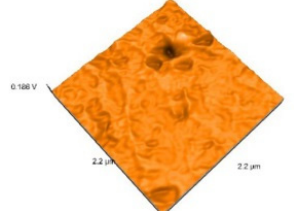

(c)

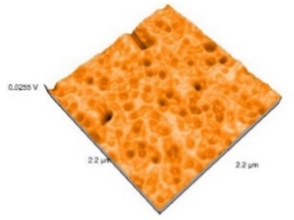

(g)

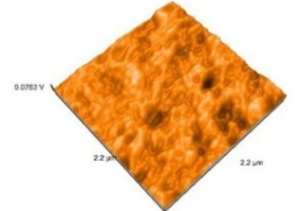

(d)

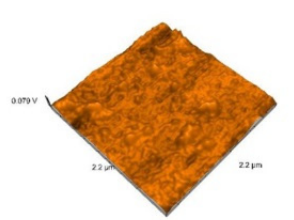

(h)

Figure 6. 3D images of Shofu HC specimens: (a) Shofu tribochemical silica airborne particle abrasion, (b) Shofu sandblasted, (c) Shofu etched, (d) Shofu polished (e) Shofu tribochemical silica airborne particle abrasion and glazed, (f) Shofu sandblasted and glazed, (g) Shofu etched and glazed (h) Shofu polished and glazed. 


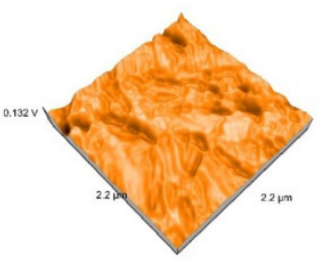

(a)

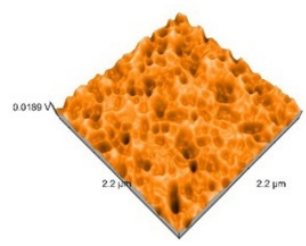

(e)

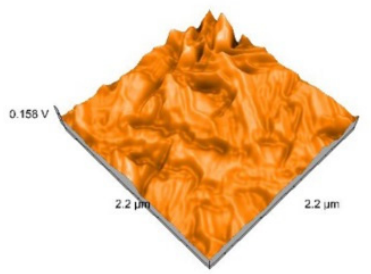

(b)

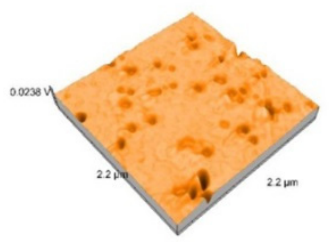

(f)

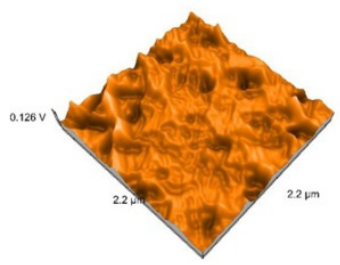

(c)

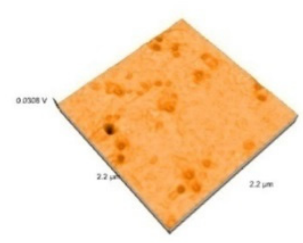

$(\mathrm{g})$

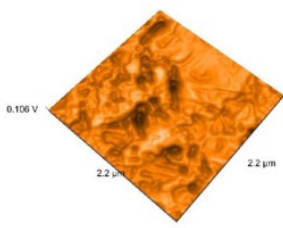

(d)

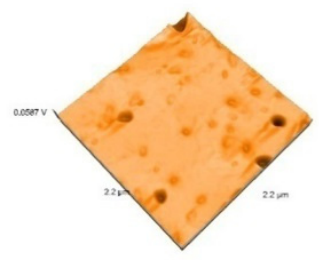

(h)

Figure 7. 3D images of Hyramic specimens: (a) Hyramic tribochemical silica airborne particle abrasion, (b) Hyramic sandblasted, (c) Hyramic etched, (d) Hyramic polished, (e) Hyramic tribochemical silica airborne particle abrasion and glazed, (f) Hyramic sandblasted and glazed, (g) Hyramic etched and glazed, (h) Hyramic polished and glazed.

\section{Discussion}

The characteristics of the material surfaces and the material compositions play an important role in obtaining favorable finishing of the surface by polishing or a thin and durable sealant layer after glazing. Therefore an improvement in bond strength is expected for microretentive surfaces and well-distributed silica content [23,24]. Because resin-composite materials combine the composition of glass ceramics and composite resins, and within this class of materials are also differences, processing procedures have to be customized. Given that there are not many such materials available on the market, surface processing investigations are welcome to be reported for each type of material.

Previous studies have suggested that pretreatments like sandblasting and tribochemical silica airborne particle abrasion are efficient in improving the bond strength of resin nanoceramic and resin-based materials [25,26]. HF etching is recommended for etchable ceramics, in order to enhance surface roughness, wettability and microretention and to reveal hydroxyl groups, which favors the chemical bonding with monomers [27].

Studies reported that the effect of HF etching on the repair bond strength for resin nanoceramics was not as high as that of sandblasting and tribochemical silica airborne particle abrasion, but more similar for hybrid ceramics [28]. Other investigations show that for the resin nanoceramics the treatment with $\mathrm{Al}_{2} \mathrm{O}_{3}$ was statistically higher than that with CoJet, followed by HF. For the polymer infiltrated ceramics (Vita Enamic), the airborne-particle abrasion techniques $\left(\mathrm{Al}_{2} \mathrm{O}_{3}\right.$ or CoJet) have been reported to be ineffective and the best results were obtained with $\mathrm{HF}(9 \%, 60 \mathrm{~s})$ followed by silane application [29,30]. Another study concluded that surface blasting followed by one layer of glaze is the best method to condition Vita Enamic [31]. Another study concluded that using 5\% hydrofluoric acid for aproximatly $60 \mathrm{~s}$ is one of the best treatments in order to increase adhesion of the characterization layer to hybrid ceramic [32].

The investigations of resin matrix ceramic materials within this study, related to the conditioning method led to a very strong correlated conditioning behavior of resin nanoceramic materials, like Lava, Cerasmart and Shofu HC samples. For these materials the microroughness decreased thus sandblasting > tricochemical conditioning $>$ etching, with a significant variation between sandblasting and etching. Previous materials are moderate correlated with Hyramic, another resin ceramic material, to which in addition sandblasting and tribochemical conditioning are close together and are moderate negative correlated to Enamic, a polymer infiltrated ceramic material, where etching is more efficient, 
resulting that all the three investigated conditioning methods lead to close microroughness values.

The topography of the surfaces was investigated by three-dimensional image generations of the surface samples, indicating visible grain boundaries after conditioning, for all materials. Nanoroughness values were strong correlated for Lava and Hyramic, with values as following: tribochemical conditioning $>$ sandblasting $>$ etching, but the values were not significantly different, valid for other materials as well. Significant higher values were recorded just for sandblasting, related to etching, for the nanoamplitude of heights.

In the literature the most frequent investigated CAD-CAM polymer infiltrated ceramic material was Vita Enamic (VITA Zahnfabrik), and the most frequent investigated resin nanoceramics were Lava Ultimate (3M ESPE) and Cerasmart (GC America). Among the types of surface-conditioning methods airborne-particle abrasion techniques with $\mathrm{Al}_{2} \mathrm{O}_{3}$ $(50 \mu \mathrm{m})$ and CoJet $30 \mu \mathrm{m}$ (dry and wet), grinding with a rotary instrument or, conditioning with HF (10\%) followed by silane application, adhesive systems, or combinations of these techniques were used. The studies compared different treatments in the same material and found different results $[33,34]$.

Other studies investigated the surface conditioning for adhesive repairs, in order to obtain optimal conditioning, related to surface finishing after glazing and associated with the best optical properties. Although various repair systems based on different conditioning protocols are available on the dental market, it is challenging for clinicians to choose the most appropriate system that would provide a reliable outcome [35]. Likewise, the optimal surface processing is a challenge for these newly developed materials and for which in vivo tests do not keep up.

After surface finishing, all the studied samples registered $R_{a}$ values below $0.2 \mu \mathrm{m}$, generally accepted to be the cut-off value [36,37]. $R_{a}$ and $R_{z}$ parameters describe the surface roughness of the samples at a micro level, and $S_{a}$ and $S_{y}$ correspond to nanoroughness. In order to have a complex view of the samples' behavior, micro and nanoroughness should be evaluated.

Polished, respective polished and glazed surfaces show significant lower microroughness values for Enamic and Shofu HC materials, compared to sandblasted or tribochemical conditioned and glazed ones. Finishing by polishing was proved to be a good choice for all materials taken into consideration, polishing and glazing likewise, excepting Hyramic. It could be concluded that for Enamic and Shofu HC sandblasted or tribochemical conditioned and glazing and for Hyramic polishing and glazing are not the best options, even surface roughness values are within clinically acceptable limits. Thereby the second null hypothesis "there are no differences in surface characteristics between different finishing protocols" was also rejected.

The investigations on nanolevel using AFM analyses demonstrated higher $S_{a}$ and $S_{y}$ values for polished surfaces for Enamic, Cerasmart and Hyramic samples, which indicates the choice of glaze, associated with the adequate conditioning method. For the other finishing methods $S_{a}$ values are similar and between 0.52 and $3.80 \mathrm{~nm}$, and for $S_{y}$ between 6.57 and $85.22 \mathrm{~nm}$.

Previously problems related to the sealants consist of low abrasion resistance, weak retention to the applied material, poor surface quality, and microcracks [38,39]. Further studies should be performed in order to investigate if surface conditioning before glazing increases the bond strength between the resin-composite CAD-CAM and the sealant, after aging procedures because resin-composite are expected to be hydrophilic [40-47]. In connection with supposed color alterations, a subsequent challenge is to evaluate the changes in optical properties of resin-composite ceramics after aging, related to surface conditioning and finishing. The study limitations were that only one type of glaze was used and that it was held in vitro and not in vivo. 


\section{Conclusions}

Within the limitations of the study given the small number of developed resincomposite materials, the following conclusions can be drawn:

1. Related to the conditioning method, Lava, Cerasmart and Shofu HC samples demonstrated a very strong correlated conditioning behavior.

2. For all tested conditioning and finishing methods, resin-composite surface roughness values were within clinically acceptable limits.

3. Finishing by polishing was proved to be a good choice for all materials taken into consideration.

4. For Enamic and Shofu HC sandblasting or tribochemical conditioning and glazing and for Hyramic polishing and glazing are not the best options, related to nanoroughness values, even microroughness values are within clinically acceptable limits.

Author Contributions: Conceptualization, L.P., S.D.P.; methodology, L.P.; software, L.P. validation, L.P.; formal analysis, L.P., M.I.B.; investigation, L.P., R.D.V.; resources, L.P.; writing-original draft preparation, L.P.; writing-review and editing, L.P., R.D.V., supervision, L.P. project administration, L.P. All authors have read and agreed to the published version of the manuscript.

Funding: This research received no external funding.

Institutional Review Board Statement: Not applicable.

Informed Consent Statement: Not applicable.

Data Availability Statement: The data presented in this study are available on request from the corresponding author.

Conflicts of Interest: The authors declare no conflict of interest.

\section{References}

1. Awada, A.; Nathanson, D. Mechanical properties of resin-ceramic CAD/CAM restorative materials. J. Prosthet. Dent. 2015, 114, 587-593. [CrossRef]

2. Zhi, L.; Bortolotto, T.; Krejci, I. Comparative in vitro wear resistance of CAD/CAM composite resin and ceramic materials. J. Prosthet. Dent. 2015, 115, 199-202. [CrossRef] [PubMed]

3. Kim, S.-H.; Choi, Y.-S.; Kang, K.-H.; Att, W. Effects of thermal and mechanical cycling on the mechanical strength and surface properties of dental CAD-CAM restorative materials. J. Prosthet. Dent. 2021. [CrossRef]

4. Porto, T.; Roperto, R.C.; Akkus, A.; Akkus, O.; Teich, S.; Faddoul, F.F.; Porto-Neto, S.; Campos, E. Effect of thermal cycling on fracture toughness of CAD/CAM materials. Am. J. Dent. 2018, 31, 205-210. [PubMed]

5. Venturini, A.B.; Prochnow, C.; Pereira, G.K.; Segala, R.D.; Kleverlaan, C.J.; Valandro, L.F. Fatigue performance of adhesively cemented glass-, hybrid- and resin-ceramic materials for CAD/CAM monolithic restorations. Dent. Mater. 2019, 35, 534-542. [CrossRef] [PubMed]

6. Ruse, N.; Sadoun, M. Resin-composite Blocks for Dental CAD/CAM Applications. J. Dent. Res. 2014, 93, 1232-1234. [CrossRef] [PubMed]

7. Porojan, L.; Vasiliu, R.D. Surface Characterization and Optical Properties of Reinforced Dental Glass-Ceramics Related to Ar-tificial Aging. Molecules 2020, 25, 3407. [CrossRef] [PubMed]

8. Vasiliu, R.D.; Porojan, L. The Effect of Thermocycling and Surface Treatments on the Surface Roughness and Microhardness of Three Heat-Pressed Ceramics Systems. Crystals 2020, 10, 160. [CrossRef]

9. Lauvahutanon, S.; Takahashi, H.; Shiozawa, M.; Iwasaki, N.; Asakawa, Y.; Oki, M.; Finger, W.J.; Arksornnukit, M. Mechanical properties of composite resin blocks for CAD/CAM. Dent. Mater. J. 2014, 33, 705-710. [CrossRef]

10. Vasiliu, R.D.; Porojan, S. Effect of thermocycling, surface treatments, and microstructure on the optical properties and rough-ness of cad-cam and heat-pressed glass-ceramics. Materials 2020, 13, 381. [CrossRef]

11. Vasiliu, R.; Porojan, S. In Vitro Study of Comparative Evaluation of Marginal and Internal Fit between Heat-Pressed and CAD-CAM Monolithic Glass-Ceramic Restorationsafter Thermal Aging. Materials 2020, 13, 4239. [CrossRef]

12. Jandt, K.D.; Watts, D.C. Nanotechnology in dentistry: Present and future perspectives on dental nanomaterials. Dent. Mater. 2020, 36, 1365-1378. [CrossRef] [PubMed]

13. Omanović-Mikličanin, E.; Badnjević, A.; Kazlagić, A.; Hajlovac, M. Nanocomposites: A brief review. Health Technol. 2020, 10, 51-59. [CrossRef]

14. Rüttermann, S.; Trellenkamp, T.; Bergmann, N.; Raab, W.H.-M.; Ritter, H.; Janda, R. A new approach to influence contact angle and surface free energy of resin-based dental restorative materials. Acta Biomater. 2011, 7, 1160-1165. [CrossRef] 
15. Flury, S.; Peutzfeldt, A.; Lussi, A. Influence of surface roughness on mechanical properties of two computer-aided design/computer-aided manufacturing (CAD/CAM) ceramic materials. Oper. Dent. 2012, 37, 617-624. [CrossRef]

16. Kaizera, M.R.; Oliveira-Ogliaria, A. Do nanofill or submicron composites show improved smoothness and gloss? A systematic review of in vitro studies. Dent Mater 2014, 30, e41-e78. [CrossRef] [PubMed]

17. Tekçe, N.; Pala, K.; Tuncer, S.; Demirci, M. The effect of surface sealant application and accelerated aging on posterior restorative surfaces: An SEM and AFM study. Dent. Mater. J. 2017, 36, 182-189. [CrossRef]

18. Heintze, S.D.; Cavalleri, A.; Forjanic, M.; Zellweger, G.; Rousson, V. Wear of ceramic and antagonist-A systematic evaluation of influencing factors in vitro. Dent. Mater. 2008, 24, 433-449. [CrossRef] [PubMed]

19. Tribst, J.P.; Dal Piva, A.M.; Werner, A.; Anami, L.C.; Bottino, M.A.; Kleverlaan, C.J. Durability of staining and glazing on a hybrid ceramics after the three-body wear. J. Mech. Behav. Biomed. Mater. 2020, 109, 103856. [CrossRef] [PubMed]

20. Tribst, J.P.M.; Dal Piva, A.M.O.; Werner, A.; Silva, L.T.S.; Anami, L.C.; Bottino, M.A.; Kleverlaan, C.J. Effect of surface treatment and glaze application on shade characterized resin-modified ceramic after toothbrushing. J. Prosthet. Dent. 2021, 125, 691.e1-691.e7. [CrossRef] [PubMed]

21. Atalay, S.; Çakmak, G.; Fonseca, M.; Schimmel, M.; Yilmaz, B. Effect of thermocycling on the surface properties of CAD-CAM ceramics after different surface treatments. J Mech Behav Biomed Mater. 2021, 121, 104646. [CrossRef]

22. Tekçe, N.; Fidan, S.; Tuncer, S.; Kara, D.; Demirci, M. The effect of glazing and aging on the surface properties of CAD/CAM resin blocks. J. Adv. Prosthodont. 2018, 10, 50-57. [CrossRef]

23. Kakaboura, A.; Fragouli, M.; Rahiotis, C.; Silikas, N. Evaluation of surface characteristics of dental composites using profilometry, scanning electron, atomic force microscopy and gloss-meter. J. Mater. Sci. Mater. Med. 2007, 18, 155-163. [CrossRef] [PubMed]

24. Tholt, B.; Miranda-Junior, W.G. Surface roughness in ceramics with different finishing techniques using atomic force microscope and profilometer. Oper. Dent. 2006, 31, 442-449. [CrossRef] [PubMed]

25. Minami, H.; Hori, S.; Kurashige, H.; Murahara, S.; Muraguchi, K.; Minesaki, Y.; Tanaka, T. Effects of Thermal Cycling on Surface Texture of Restorative Composite Materials. Dent. Mater. J. 2007, 26, 316-322. [CrossRef]

26. Jones, C.S.; Billington, R.W.; Pearson, G.J. The in vivo perception of roughness of restorations. Br. Dent. J. 2004, 196, 42-45. [CrossRef]

27. Nguyen, J.-F.; Migonney, V.; Ruse, N.D.; Sadoun, M. Resin composite blocks via high-pressure high-temperature polymerization. Dent. Mater. 2012, 28, 529-534. [CrossRef] [PubMed]

28. Sideridou, I.D.; Karabela, M.M. Sorption of water, ethanol or ethanol/water solutions by light-cured dental dimethacrylate resins. Dent. Mater. 2011, 27, 1003-1010. [CrossRef]

29. Horvath, S.D. Key Parameters of Hybrid Materials for CAD/CAM-Based Restorative Dentistry. Compend. Contin. Educ. Dent. 2016, 37, 638-643. [PubMed]

30. Alamoush, R.A.; Silikas, N.; Salim, N.; Al-Nasrawi, S.; Satterthwaite, J.D. Effect of the Composition of CAD/CAM Composite Blocks on Mechanical Properties. BioMed Res. Int. 2018, 2018, 4893143. [CrossRef]

31. Köroğlu, A.; Sahin, O.; Dede, D.; Yilmaz, B. Effect of different surface treatment methods on the surface roughness and color stability of interim prosthodontic materials. J. Prosthet. Dent. 2015, 115, 447-455. [CrossRef]

32. Sahin, O.; Koroglu, A. Effect of surface sealant agents on the surface roughness and color stability of denture base materials. J. Prosthet. Dent. 2016, 116, 610-616. [CrossRef]

33. Pereira, G.; Venturini, A.; Silvestri, T.; Dapieve, K.; Montagner, A.; Soares, F.; Valandro, L. Low-temperature degradation of Y-TZP ceramics: A systematic review and meta-analysis. J. Mech. Behav. Biomed. Mater. 2015, 55, 151-163. [CrossRef] [PubMed]

34. Marshall, S.J.; Bayne, S.C. A review of adhesion science. Dent. Mater. 2010, 26, 11-16. [CrossRef] [PubMed]

35. Alp, G.; Subaşı, M.G.; Johnston, W.M.; Yilmaz, B. Effect of different resin cements and surface treatments on the shear bond strength of ceramic-glass polymer materials. J. Prosthet. Dent. 2018, 120, 454-461. [CrossRef]

36. Arpa, C.; Ceballos, L.; Fuentes, M.V.; Perdigão, J. Repair bond strength and nanoleakage of artificially aged CAD-CAM composite resin. J. Prosthet. Dent. 2019, 121, 523-530. [CrossRef]

37. Wu, X.; Xie, H. Effect of tribochemical silica coating or multipurpose products on bonding performance of a CAD/CAM resin-based material. J. Mech. Behav. Biomed. Mater. 2019, 90, 417-425. [CrossRef]

38. Ramakrishnaiah, R.; Alkheraif, A.A.; Divakar, D.D.; Matinlinna, J.P.; Vallittu, P.K. The Effect of Hydrofluoric Acid Etching Duration on the Surface Micromorphology, Roughness, and Wettability of Dental Ceramics. Int. J. Mol. Sci. 2016, 17, 822. [CrossRef] [PubMed]

39. Porojan, L.; Vasiliu, R.D. Surface Quality Evaluation of Removable Thermoplastic dental Appliances Related to Staining Beverages and Cleaning Agents. Polymers 2020, 12, 1736. [CrossRef]

40. Sismanoglu, S.; Yildirim-Bilmez, Z.; Erten-Taysi, A.; Ercal, P. Influence of different surface treatments and universal adhesives on the repair of CAD-CAM composite resins: An in vitro study. J. Prosthet. Dent. 2020, 124, 238.e1-238.e9. [CrossRef]

41. Veríssimo, A.H.; Moura, D.M.D. Effect of different repair methods on the bond strength of resin composite to CAD/CAM materials and microorganisms adhesion: An in situ study. J. Dent. 2020, 93, 103-166. [CrossRef] [PubMed]

42. Güngör, M.B.; Nemli, S.K. Effect of surface treatments on shear bond strength of resin composite bonded to CAD/CAM resin-ceramic hybrid materials. J. Adv. Prosthodont. 2016, 8, 259-266. [CrossRef] [PubMed] 
43. Moura, D.M.D.; Veríssimo, A.H.; Vila-Nova, T.E.L.; Calderon, P.S.; Özcan, M.; Souza, R.O.A. Which surface treatment promotes higher bond strength for the repair of resin nanoceramics and polymer-infiltrated ceramics? A systematic review and metaanalysis. J. Prosthet. Dent. 2021. [CrossRef]

44. Elsaka, S.E. Repair bond strength of resin composite to a novel CAD/CAM hybrid ceramic using different repair systems. Dent. Mater. J. 2015, 34, 161-167. [CrossRef]

45. Blum, I.R.; Nikolinakos, N.; Lynch, C.D.; Wilson, N.H.; Millar, B.J.; Jagger, D.C. An in vitro comparison of four intra-oral ceramic repair systems. J. Dent. 2012, 40, 906-912. [CrossRef] [PubMed]

46. Sahin, O.; Dede, D.Ö. Influence of surface sealant agents on the surface roughness and color stability of artificial teeth. J. Prosthet. Dent. 2015, 114, 130-137. [CrossRef] [PubMed]

47. Grangeiro, M.T.V.; Rossi, N.R.; Barreto, L.A.L.; Bottino, M.A.; Tribst, J.P.M. Effect of Different Surface Treatments on the Bond Strength of the Hybrid Ceramic Characterization Layer. J. Adhes. Dent. 2021, 23, 429-435. [CrossRef] 